\title{
Agôn
}

Revue des arts de la scène

HS 2 | 2014

Mettre en scène le conte

\section{Entretien avec Antonin Leymarie, compositeur}

\section{Antonin Leymarie et Marion Boudier}

\section{(2) OpenEdition}

Journals

Édition électronique

URL : https://journals.openedition.org/agon/3071

DOI : 10.4000/agon.3071

ISSN : 1961-8581

Éditeur

Association Agôn

Référence électronique

Antonin Leymarie et Marion Boudier, « Entretien avec Antonin Leymarie, compositeur », Agôn [En ligne], HS 2 | 2014, mis en ligne le 13 juillet 2014, consulté le 18 juin 2021. URL : http:// journals.openedition.org/agon/3071; DOI : https://doi.org/10.4000/agon.3071

Ce document a été généré automatiquement le 18 juin 2021.

Association Agôn et les auteurs des articles 


\title{
Entretien avec Antonin Leymarie, compositeur
}

\author{
Antonin Leymarie et Marion Boudier
}

La musique de Cendrillon est constituée en majorité de compositions d'Antonin Leymarie. À celles-ci s'ajoutent d'autres matières sonores et quelques airs connus (notamment Father and son de Cat Stevens) plus ou moins retravaillés ${ }^{1}$.

Antonin Leymarie est interprète, compositeur et batteur. Après une formation de percussionniste classique au conservatoire du $10^{\mathrm{e}}$ arrondissement à Paris où il a également étudié le piano, il a travaillé les percussions mandingues et maliennes avant de se dédier à la batterie. C'est en tant que batteur et compositeur qu'il a collaboré pour la première fois avec Joël Pommerat pour Cet enfant (2006 - recréation de Qu'est-ce qu'on a fait ?, 2003). Depuis Pinocchio (2008), il a composé de la musique pour tous les spectacles de l'auteur-metteur en scène.

Antonin Leymarie évolue également avec plusieurs groupes, notamment La Compagnie Imperial, Le Magnetic Ensemble, le Surnatural Orchestra et il joue en live avec le cirque Les Colporteurs depuis une quinzaine d'années.

Antonin LEYMARIE - Je travaille avec l'image depuis longtemps, au cirque. J'ai rencontré Joël sur Cet enfant. J'avais vu quelques spectacles avant, car mon père (François Leymarie) travaillait déjà avec la compagnie. J'étais fasciné par l'univers sonore. Mais jusqu'à Cet enfant, il y a une utilisation assez parcimonieuse des mélodies. Avec ce spectacle, Joël a réussi un tour de passe-passe exceptionnel avec la musique, avec le tulle et les instruments derrière. La musique est enregistrée : il n'y a pas de musiciens sur scène, ce sont les comédiens qui manipulent les instruments. Mais grâce au travail de sonorisation de François et Grégoire (Leymarie), le public se plaint toujours que les musiciens ne viennent pas saluer à la fin du spectacle ! 


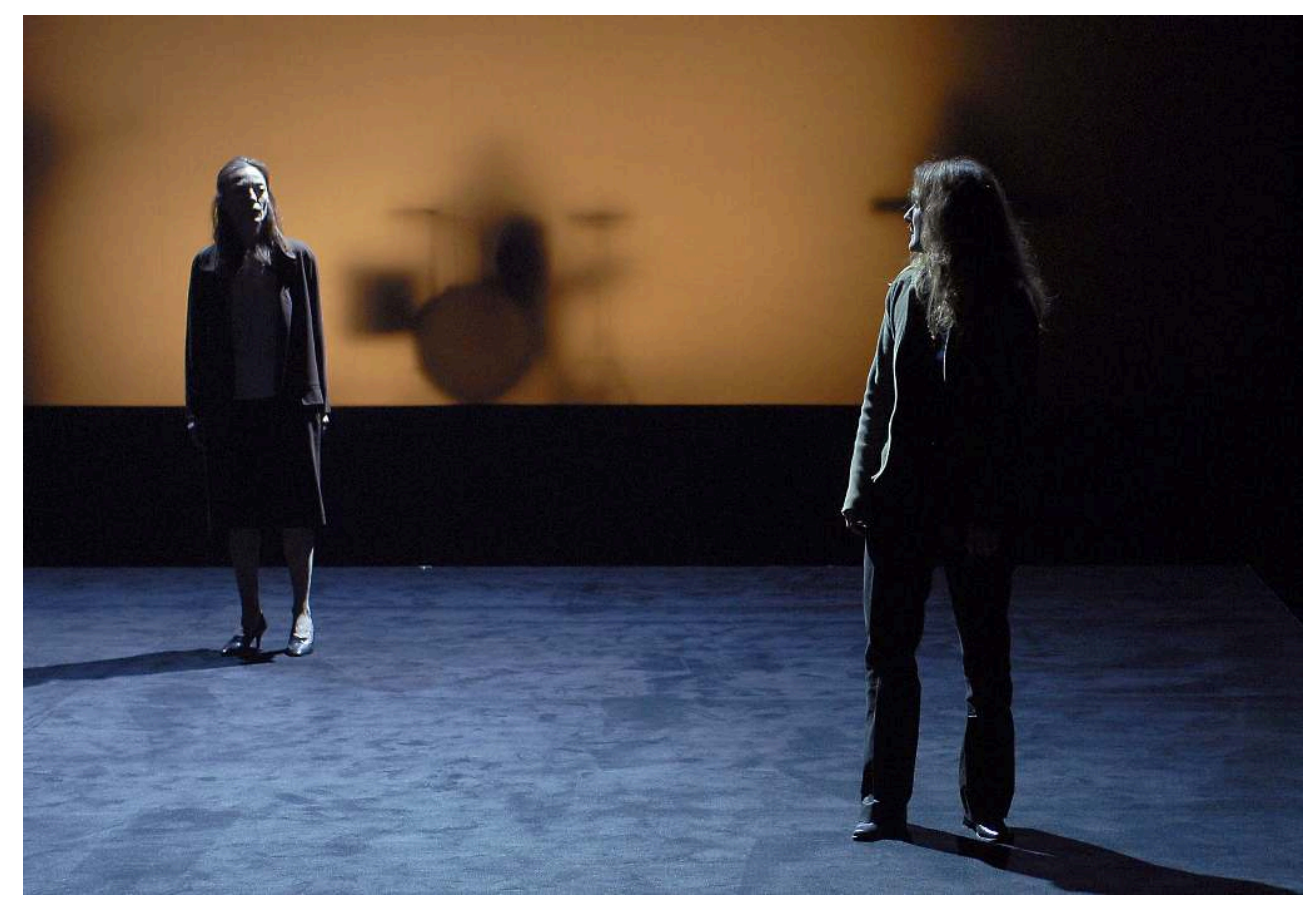

(c) Ramon Senera

Marion BOUdieR - Comment composes-tu tes musiques pour les spectacles de Joël : est-ce par rapport au texte, à des thèmes ou pour des scènes auxquelles tu assistes en répétition par exemple?

A. L. - Ni l'un ni l'autre. En général, j'ai un rendez-vous avec Joël, avant les répétitions quand il n'a pas encore écrit. Il a déjà une idée de ce qui va se passer, donc il m'en parle mais sans trop me révéler de choses. Il me laisse beaucoup d'ouverture pour les propositions que je peux lui faire. C'est sa manière de travailler, pas seulement avec la musique. On ne travaille jamais ensemble sur une scène en particulier - c'est arrivé une fois sur Je tremble, mais ça n'avait pas fonctionné. Joël se nourrit de ce que son équipe propose, et la musique on doit la faire avant. J'ai un budget de recherche et un rapport de confiance avec Joël. On ne fonctionne pas à la minute - heureusement que Joël ne fonctionne pas comme cela! Il me fait confiance pour produire une masse dans laquelle il y aura des choses qui lui parleront. C'est pareil pour la lumière, pour l'univers sonore, etc. Je sais que ce que je propose pourra être bouclé, mixé, transformé. Dans Pinocchio par exemple, il y a énormément de morceaux qui se développent avec des harmonies, et très souvent c'est le début qui est repris en boucle. C'est Joël qui fait la partition musicale finalement.

M. B. - Est-ce que certaines scènes s'écrivent à partir de la musique?

A. L. - Au début de la recherche, il y a toujours de la musique, de même qu'il y a de la lumière et des costumes. Tout inspire tout. Je travaille en amont pour ce moment. Sinon, je ne sais pas comment il trouve le temps, mais Joël écoute aussi beaucoup de musique. Il écrit souvent en écoutant de la musique, que ce soit la mienne ou d'autres musiques qu'on lui conseille ou qu'il choisit seul. 
M. B. - A quel moment du processus de création commences-tu à faire des propositions musicales concrètes?

A. L. - Je viens un peu au début des phases d'expérimentation, mais assez peu, car il me faut le temps d'écrire la musique : y réfléchir, trouver les sons, les instruments, les musiciens, puis les enregistrer. Toute cette recherche ne peut pas se faire pendant les phases de création. Je travaille donc un peu en parallèle. Pour intervenir pendant la période de création, il faudrait que je fasse de la musique en solitaire, sur ordinateur par exemple. Je ne fais pas de recherche en directe, car je travaille en acoustique, en lien avec des musiciens et avec des mélodies. Il nous est déjà arrivé de travailler en live en répétition. Mais au théâtre, la base du travail reste le texte, ce qui contraint beaucoup le volume des musiciens.

M. B. - Pour Cendrillon, est-ce que cela signifie que tu n'es venu que pour la dernière phase de création à Bruxelles?

A. L. - Non, j'étais passé un peu à Châteauvallon quelques mois avant. Puis j'ai envoyé des choses à Joël pour qu'il les essaye avec l'équipe. A partir des retours, je peux proposer autre chose, etc. Quand je suis présent aux répétitions, j'essaye de ne pas trop perturber le travail en cours, car j'arrive dans un processus lent et long. Sur Cendrillon, Joël m'avait donné ses notes. On ne travaille pas toujours comme cela. J'ai bien aimé, et en conséquence j'ai essayé de travailler sur des thématiques par personnages. J'ai notamment fait une grande recherche sonore autour du cristal, pour la maison de verre au premier degré, mais surtout pour trouver une fragilité du personnage de Sandra. J'ai contacté Thomas Bloch qui joue de trois instruments très rares : de l'onde Martenot, du cristal Baschet et du glassharmonica (cf. extrait sonore en annexe $e^{2}$. On a fait une séance avec lui et un guitariste. Mais si Joël veut une musique baroque, par exemple pour le moment de l'arrivée au bal, je ne vais pas imiter ce style.

\section{B. - Comment se passe la collaboration avec Grégoire et François Leymarie?}

A. L. - François est responsable du son pour la compagnie depuis une vingtaine d'années. Il fait les décors sonores, c'est-à-dire qu'il travaille sur des matières sonores et leur diffusion. Il passe beaucoup de temps à trouver et enregistrer des sons, à les remanier, et à trouver comment les diffuser dans la salle. Cet univers sonore très riche et la qualité de sa diffusion sont propres au travail de Joël; c'est une de ses demandes depuis le début. François est présent en création. Il crée l'univers et doit gérer toutes les questions de diffusions selon les scénographies. Grégoire est entré dans la compagnie il y a dix ans : il travaille sur la diffusion du son au sens large et sur la création de boucles ou transformations en direct à partir des sources qu'on lui apporte. Pendant les phases de création, il fait un énorme travail de mélange des différentes sources. C'est lui qui fait cette "mixture ». Pour La Réunification des deux Corées, il m'a vraiment impressionné ; je pense que les gens qui s'intéressent au son devraient venir voir ces moments-là si c'était possible. En ce qui me concerne, pour résumer le fonctionnement de ma contribution : j'ai une commande sur une création, j'imagine les instruments, j'écris, je trouve les instrumentistes. Puis il faut gérer les disponibilités de tout le monde pour faire des séances d'enregistrement, d'un ou deux jours en général. C'est François qui enregistre, puis on mixte ensemble. On livre une sélection à Joël qui choisit des musiques que Grégoire et François vont encore retravailler. Dans Cendrillon, il y a énormément de moments de mes musiques qui sont repris en boucles, par exemple. 
M. B. - Que deviennent les morceaux coupés ou laissés de côté ? Est-ce que tu les reprends avec tes groupes?

A. L. - La dernière chanson de Cendrillon, je l'avais composée pour Cercles/Fictions. Tout ce que j'ai fait appartient à la compagnie, qui a produit les enregistrements. Cela veut dire qu'on a plusieurs années de musique dans lesquelles on peut piocher. Autour de Cercles/Fictions, on a fait une soirée de concert à l'Atelier du Plateau. C'était ce que j'appelle "la musique du cercle», pas spécialement celle du spectacle. La musique avant qu'elle soit élaguée. J'espère qu'on pourra refaire ce genre de soirée, peut-être en partant de musiques de La Grande et Fabuleuse Histoire du commerce et de La Réunification des deux Corées, avec des cordes... Faire un concert dans un lieu de théâtre comme l'Atelier du Plateau, c'est quelque chose qui me plaît beaucoup. Ce que je préfère en fait, c'est les concerts, c'est jouer. Cela m'enrichit pour écrire et réaliser de la musique. La vraie vie, c'est être sur scène. Mais les deux activités s'enrichissent mutuellement.

M. B. - Est-ce que le fait que Cendrillon soit un spectacle tout public mais tout de même à destination de jeunes spectateurs t'a influencé pour composer la musique?

A. L. - Oui, d'ailleurs avec Joël, on n'a pas tout de suite été raccord sur ce point ! Comme je savais que ce serait un spectacle pour enfants mais que sa Cendrillon était très dark, je pensais qu'il fallait, comme dans Pinocchio, révéler un peu de simplicité et de naïveté. Au début, j'ai un peu trop joué la carte des consonances et de la simplicité musicale. Pour Pinocchio, la musique allège beaucoup un texte et une mise en scène qui peuvent sembler durs pour des enfants. Sur Cendrillon, après les remarques de Joël, j'ai ajouté une séance de clarinette (Gérald Chevillon) pour rompre les consonances : il y a un thème à la clarinette basse qu'on a réenregistré avec la moitié de la clarinette démontée pour travailler sur des matières dissonantes. Je me garde toujours du temps, de sorte que Joël sait qu'il peut m'appeler dans les dernières semaines s'il lui manque quelque chose.

M. B. - Dans le texte publié, qui est selon Joël une «trace du spectacle », il n'y pas d'indications musicales dans les didascalies hormis la référence à Father and son de Cat Stevens (la chanson du Prince). Qu'en penses-tu?

A. L. - C'est une question que je ne me pose pas trop. Par rapport à l'idée que les didascalies pourraient servir à une mise en valeur de certains aspects du travail spectaculaire, je coupe court tout de suite. La mise en valeur du travail a lieu au moment où ça travaille, pendant la création, et puis il y a aussi une part de réalisation personnelle, individuelle pour chaque membre de l'équipe. On a envie de bien travailler, il n'y a pas d'égo. Par rapport à l'édition, on pourrait faire un livre musical, avec un $\mathrm{CD}$, ça serait autre chose...

\section{NOTES}

1. Voir la liste des musiques originales et additionnelles publiée dans Joël Pommerat - Cendrillon, Christophe Triau, Scérén CNDP-CRDP, coll. Arts au singulier Théâtre, 2014, p. 67. 
2. Extraits sonores en annexe: "Th Cendrillon1 onde cello", duo onde Martenot et guitare finalement absent du spectacle ; «Cercles 62_01 tutti », musique de la fin du spectacle. 